\title{
Circuit
}

Musiques contemporaines

CIRCUIT

\section{L’odyssée musicale afrocubaine}

\section{Entre art et politique, entre nationalisme et panaméricanisme}

An Afro-Cuban Musical Odyssey

Between Art and Politics, Nationalism and Panamericanism

\section{Leiling Chang}

Volume 17, numéro 2, 2007

Plein sud : Avant-gardes musicales en Amérique latine $a u x^{\mathrm{e}}$ siècle

URI : https://id.erudit.org/iderudit/016843ar

DOI : https://doi.org/10.7202/016843ar

Aller au sommaire du numéro

Éditeur(s)

Les Presses de l'Université de Montréal

ISSN

1183-1693 (imprimé)

1488-9692 (numérique)

Découvrir la revue

Citer cet article

Chang, L. (2007). L’odyssée musicale afrocubaine : entre art et politique, entre nationalisme et panaméricanisme. Circuit, 17(2), 93-106.

https://doi.org/10.7202/016843ar

\section{Résumé de l'article}

À Cuba, les premières décennies $d u x^{e}$ siècle montrent un contexte social et culturel des plus intéressants pour l'historien, ceci dû à plusieurs facteurs : la société cubaine affronte une nouvelle situation démographique après l'abolition de l'esclavage et le conséquent déplacement de la population africaine esclave vers les villes ; la naissance de la république de Cuba, à la fin de la colonisation espagnole, est marquée par une forte dépendance des États-Unis ; des mouvements d'avant-garde politique et culturelle mènent une lutte sans répit contre les esprits les plus traditionalistes; le monde politique est des plus controversés, entre la corruption, l'assassinat des étudiants universitaires, la présence de militaires américains, entre autres aspects. Dans ce contexte a lieu l'un des plus beaux mouvements artistiques de l'histoire de Cuba : l'afrocubanisme. Ce mouvement, cherchant à revendiquer l'origine africaine de la nation cubaine, est en même temps une quête de modernité accrue et sera le point de départ de l'art cubain $\mathrm{du} \mathrm{xx}^{\mathrm{e}}$ siècle. Parmi les figures qui marquent ce mouvement, Amadeo Roldán, compositeur, en est le pionnier et le divulgateur. Dans ses oeuvres, comme dans sa poétique musicale, nous trouvons l'origine de la musique cubaine $\mathrm{du} \mathrm{xx}^{\mathrm{e}}$ siècle, jusqu'à nos jours, ainsi que l'évidence d'une lutte plus large, panaméricaine, qui cherche modernité et universalité à partir des recherches folkloriques.
Ce document est protégé par la loi sur le droit d'auteur. L'utilisation des services d’Érudit (y compris la reproduction) est assujettie à sa politique d'utilisation que vous pouvez consulter en ligne.

https://apropos.erudit.org/fr/usagers/politique-dutilisation/ 


\title{
L’odyssée musicale afrocubaine
}

\author{
Entre art et politique, entre nationalisme et panaméricanisme
}

Leiling Chang

Découverte et colonisée par l'Espagne, l'île de Cuba ne vit l'éveil d'une conscience nationale et d'un désir d'indépendance qu'au XIX siècle, période de laquelle émerge la figure du criollo, cet homme qui éprouve déjà un attachement patriotique pour sa terre natale et sent se défaire les liens affectifs avec la métropole espagnole. Le criollo est déjà conscient de son identité culturelle, ainsi que de sa différence avec les générations précédentes; puisqu'il se sent fier de sa culture, la colonisation devient un mal intolérable. Parmi les multiples positions politiques des criollos du XIX siècle, qui allaient de l'annexionnisme aux États-Unis à l'indépendance totale du pays, c'est sans doute cette dernière qui encourage les esprits les plus fougueux et mène à des luttes armées pour l'indépendance (1868-1878; 1895-1898). Simultanément, naissent un art nationaliste et une musique nationaliste issue d'une modification organologique, mélodique et rythmique des danses européennes. À partir des textes de Fernando Ortiz (1881-1969), ethnologue et anthropologue cubain du début du $\mathrm{Xx}^{\mathrm{e}}$ siècle, cette transformation de l'héritage européen est souvent nommée "processus de transculturation ${ }^{1}$ ", terme qui cherche à exprimer l'enchevêtrement des cultures européennes et africaines, en même temps que la nouveauté et l'originalité du produit culturel qui en résulte. Dans le cas spécifique de la musique, les bals de salon de la bourgeoisie criolla du XIX siècle constituent de vrais lieux de métissage : le métier de musicien étant réservé
1. Ce terme a été appliqué à d'autres pays de l'Amérique latine puisqu'il résume bien les caractéristiques propres du métissage produit par la colonisation et l'esclavage. Or, il est souvent difficile de comprendre sa signification Iorsqu'on l'utilise dans des textes francophones, puisque la clarté et la précision qu'il possède dans la langue espagnole sont souvent absentes dans d'autres langues. Malgré ce problème, nous préférons l'utiliser tel quel pour éviter d'employer d'autres termes non équivalents. 
aux Noirs libres, les orchestres qui exécutaient des contredanses françaises, des valses et d'autres danses de l'époque deviennent les véritables artisans de la fusion criolla.

La deuxième guerre d'indépendance (1895-1898) conduit presque à l'indépendance du pays. Cuba cesse d'être une colonie de l'Espagne, mais une intervention nord-américaine, survenue juste à la fin de la guerre, marque au contraire le début d'une société «néocoloniale», en apparence libre mais largement dépendante des États-Unis. L’année 1900 marque la naissance de la République de Cuba. Au début du Xx siècle, l'échec de la dernière guerre, ajouté au statut officiel d'indépendance, n'a fait qu'intensifier le sentiment nationaliste et prolonger la quête continuelle de l'identité culturelle. La recherche d'une expression nationale devient plus sincère, moins superficielle qu'au cours du siècle précédent, en même temps qu'elle affronte une nouvelle situation démographique qui provoque l'éveil à une réalité, présente depuis plus de trois siècles, mais longtemps occultée : l'esclavage.

En effet, un siècle entier d'essor d'une conscience nationale et, dans le monde de l'art, d'une esthétique nationaliste, s'était écoulé avant que la nouvelle république n'arrive en 1900. Mais, si nous regardons de près, nous réalisons qu'en fait l'expression nationaliste du criollo du XIX ${ }^{\mathrm{e}}$ siècle prenait en considération un seul élément de la mosaïque ethnique qui compose Cuba, l'héritage européen, même s'il assume et s'identifie aux modifications de cet héritage produites par la transculturation avec la population noire. Mais, pour le criollo, cette population noire n'est autre que le faible pourcentage de Noirs libres des zones urbaines, qui exécute divers métiers, dont celui de musicien, qui est né à Cuba et ne conserve de son lieu d'origine que l'héritage transmis par ses ancêtres, affaibli par les années; c'est le Noir libre criollo, non l'esclave africain. Le véritable héritage culturel africain, gardé et cultivé dans ses formes pures - seulement transformé par la fusion entre les diverses ethnies africaines qui composaient la population esclave -, presque sans transculturation avec la culture espagnole, restait encore ignoré, pendant tout le XIX ${ }^{\mathrm{e}}$ siècle, enfermé dans les baraques d'esclaves, dans les murs clos des territoires de la canne à sucre, isolé même des zones urbaines dans lesquelles l'art se développait avec plus de rapidité. Le criollo du XIX siècle ignore donc l'esclave africain.

L'année 1900, juste quatorze ans après l'abolition officielle de l'esclavage (1886), marque alors le début d'un processus démographique d'importance : la population d'origine africaine récemment sortie des baraques se déplace vers les zones urbaines et les habite, changeant radicalement les pourcentages dans la composition ethnique des villes. Sur le plan social, ce processus confrontait toutes les couches de la société à l'évidence et l'importance - en nombre 
et en poids culturel - de cette composante ethnique et donc à l'origine africaine de la nation cubaine. La société cubaine au complet réagissait à cette nouvelle composition ethnique des villes.

La prise de position des intellectuels et des artistes du début du XX⿳亠丷厂 siècle par rapport à l'origine africaine de la nation cubaine est devenue alors l'enjeu qui déterminait les attitudes d'avant-garde ou de traditionalisme. Dans ce contexte, deux lignes de pensée s'affrontaient : l'indigénisme et l'afrocubanisme. Il était clair pour tous que l'Espagne seule ne composait absolument pas la culture cubaine; tout un siècle de pensée criolla avait démontré ce fait. Mais quel était l'autre ou les autres composantes ethniques du métissage cubain? Pour beaucoup d'intellectuels et d'artistes de l'époque, le fait même de poser cette question, ainsi que sa réponse, ne cherchait qu'à cacher le véritable enjeu : nier ou accepter la culture noire, donc, l'ascendance africaine de Cuba.

Des intellectuels renommés, accompagnés de la conviction sans détour de la bourgeoisie du moment, ont créé de toutes pièces une théorie appelée «indigéniste » axée sur une supposée survivance de la culture aborigène. Cette dernière, mélangée à l'héritage espagnol, conformerait la figure idyllique du nouveau criollo, symbolisé par le campesino (l'homme de la campagne), très proche de la race blanche. Il importait peu que des travaux scientifiques en histoire, archéologie et anthropologie aient démontré, déjà à l'époque, l'impossibilité d'une telle hypothèse (puisque la population aborigène avait disparu presque dans sa totalité déjà à la fin du Xvi siècle, environ trois siècles avant que le véritable processus de métissage se déclenche); il importait peu que la présence noire soit évidente, corresponde à un haut pourcentage de la population et soit présente dans l'île depuis le XVI e siècle. La théorie indigéniste cherchait à expulser la culture africaine de la construction identitaire qui constituait l'image de la nation au début du Xx siècle, prolongeant ainsi, tout simplement, la discrimination raciale et culturelle que le système d'esclavage avait instaurée pendant des siècles comme pratique courante dans le pays.

Un musicien, compositeur de grand talent, et qui, sans doute, a beaucoup fait, sur d'autres plans, pour le développement culturel et musical de Cuba, était au centre de cette polémique : Eduardo Sanchez de Fuentes (1874-1944). Avec l'objectif de créer une musique nationaliste nouvelle, il fonde ses recherches esthétiques sur cette incertaine origine aborigène du criollo. Le fait d'avoir consacré sa vie créatrice à l'étude des documents «aborigènes» transcrits dans les Antilles (dont l'authenticité avait déjà était démentie par des recherches scientifiques) transforme Sanchez de Fuentes en un défenseur à outrance de la théorie indigéniste. Le mensonge se prolongea pendant plusieurs décennies, constituant un véritable sentier d'engagement culturel et 
politique appuyé par les couches sociales les plus traditionalistes, effrayées devant la présence noire.

La réponse des intellectuels d'avant-garde ne se fait pas attendre. Avec une base solide, établie sur les recherches ethnologiques et anthropologiques de Fernando Ortiz, ces intellectuels et artistes d'avant-garde unissaient deux objectifs dans leurs quêtes esthétiques : défendre la présence noire en faisant de cette composante ethnique la base de la recherche du national; se mettre à jour avec les courants artistiques les plus récents en Europe, qui englobaient le mouvement surréaliste, la découverte de la richesse culturelle de l'Afrique, les tendances folkloristes et, sur le plan musical, la spéculation sur des formules rythmiques insoupçonnées, l'ouverture à des univers sonores inédits, comprenant les bruits et sonorités âcres, d'effet brusque et souvent sauvage (le Stravinsky du Sacre du Printemps constituait un paradigme), l'impact du rituel dans les ballets russes dirigés par Diaghilev, entre autres aspects.

À Cuba, redécouvrir l'Afrique signifiait plonger dans un monde musical d'une extraordinaire richesse rythmique, mélodique, organologique, religieuse, rituelle; cela signifiait aussi ouvrir une porte à un monde sonore nouveau, véritable scandale pour la bourgeoisie du moment, prise dans ses goûts arriérés, préférant la facilité et le divertissement des romances et des nocturnes en vogue, des arias d'opéras italiens bien connus et des danses de salon. Alors, l'impact du mouvement afrocubain, à la fois recherche ethnologique et quête de modernité accrue, ne surprend guère. Les musiciens et artistes d'avantgarde sentaient un grand besoin de réveiller les esprits et la lutte pour l'acceptation de cette modernité et du monde africain devait se poursuivre pendant une longue période, puisque la pensée traditionaliste a tout fait pour valider ses positions indigénistes et continuer autant la discrimination et la répugnance esthétique envers la culture africaine que ses habitudes de consommation artistiques ancrées dans le XIX ${ }^{\mathrm{e}}$ siècle, lui-même en franc retard colonial.

Mais il y avait aussi d'autres enjeux : dans ce contexte, promulguer une musique nouvelle était, pour les compositeurs, une manière de s'engager politiquement dans la lutte pour la fin de la dépendance de l'Empire américain, qui était le sceau de la nouvelle république, bien nommée «néocoloniale», instaurée au début du xxe siècle. Au contraire, adopter la théorie indigéniste équivalait à accepter la nouvelle situation de Cuba, dans laquelle l'ambassadeur américain était la figure politique la plus respectée et la protection contre les invasions américaines la seule préoccupation du gouvernement, au point de justifier l'assassinat politique constant, public et massif. Ces liens entre l'activité artistique et l'engagement politique ne sont pas métaphoriques : les individus concrets, impliqués dans chacune de ces positions opposées, prenaient 
parti dans une lutte double, à la fois esthétique et politique. Art et prise de position par rapport au monde social et aux décisions politiques n'ont jamais été si imbriqués à Cuba qu'au cours de cette période riche pendant laquelle les crises politiques accompagnent une véritable effervescence créatrice dans le monde de l'art, le tout dans un état général de fébrilité, dans un éveil éclatant des mentalités récemment sorties des structures coloniales paralysantes du siècle précédent.

Dans ce contexte social, politique et culturel complexe, Cuba vit probablement l'essor des pratiques musicales le plus impactant de son histoire, sur tous les plans possibles : la composition (naît, par exemple, le symphonisme nationaliste cubain), la divulgation, la pédagogie, la fondation de revues spécialisées, des sociétés pro-arte, les concerts d'avant-garde, la fondation des premiers orchestres symphoniques et les développement et internationalisation de la musique populaire.

\section{Les années 1923 à 1932}

La décennie qui comprend les années entre 1923 et 1932 a souvent été appelée «décennie critique»:

Découverte par Colomb au XV siècle, par les Anglais au XvIII ${ }^{\mathrm{e}}$ siècle, par Humbold et les intellectuels criollos au XIX ${ }^{\mathrm{e}}$ siècle, il ne manquait que Cuba se découvre ellemême au Xx ${ }^{\mathrm{e}}$ siècle, et c'est ce qui arriva dans cette décennie. Ce que la période de 1923 à 1932 a apporté sur les plans politique, social et culturel, fut l'esprit de l'époque, et avec cela, le besoin urgent d'une transformation². (Ambrosio, cité dans Gómez, 1977 , p. 42)

Cette nouvelle prise de conscience nationale arrive dans un contexte de crise sociale majeure. Le chômage était au maximum, puisque le monopole de l'industrie du sucre imposait son système de travail cyclique obligeant à des périodes de «temps mort ${ }^{3} »$. Les révoltes sociales se multiplient et les intellectuels s'engagent dans une lutte inspirée par la révolution russe, la révolution mexicaine, l'essor de la pensée marxiste en Amérique latine et la réforme universitaire de l'Argentine. Sur place aussi il y a des événements qui justifient ce désir de transformation : l'intervention nord-américaine, qui, depuis le début du $\mathrm{XX}^{\mathrm{e}}$ siècle, dérangeait les esprits nationalistes, devenait beaucoup plus évidente avec la présence d'autorités militaires américaines avec un fort pouvoir politique. Ensemble, ces facteurs ont produit une suite d'événements de protestation sociale dans lesquels les intellectuels, les artistes et les étudiants universitaires ont joué un rôle prépondérant : à partir de la Protesta de los Trece (Protestation des Treize) s'organise et se systématise un mouvement d'intellectuels sous la direction du poète Rubén Martinez Villena; l'ethnologue
2. Ambrosio, En blanco y negro, p. 45.

3. On appelait ainsi les périodes d'arrêt de la production de sucre. 
4. Bongó : instrument de percussion typiquement cubain, d'origine africaine, consistant en deux petits tambours unis par une planche en bois que le musicien place entre les genoux.
Fernando Ortiz dénonce publiquement la corruption politique et demande au gouvernement plus d'attention à l'éducation, l'hygiène et la santé publiques; surgit le mouvement des Vétérans et des Patriotes, organisé également pour la dénonciation de la corruption politique; les étudiants universitaires se regroupent autour de Julio Antonio Mella, directeur de la Fédération des étudiants universitaires (qui sera assassiné par le corps policier quelques années plus tard lors d'une manifestation universitaire), dans la lutte pour la transformation du corps des professeurs, la participation des étudiants à la gestion de l'université, le droit à un enseignement moderne et gratuit, accompagné de méthodes scientifiques et pour l'autonomie universitaire par rapport aux structures gouvernementales; surgit le groupe Minoriste, composé d'intellectuels (écrivains, musiciens, peintres, chercheurs) de diverses générations, dont la «Déclaration » conceptualisa la lutte contre la corruption.

Ce dernier groupe se réunissait quotidiennement à l'hôtel Lafayette, situé dans le centre de La Havane. Parmi ses membres se trouvait le poète Villena, mais aussi Alejo Carpentier (1904-1980), probablement l'écrivain cubain le plus connu dans le monde francophone. Carpentier, avec son énorme culture et son savoir, ancré à la fois dans une connaissance approfondie de l'histoire de l'Amérique latine et des Antilles et dans un rapport affectif ( à cause de son origine bretonne et de son bilinguisme) ainsi qu'intellectuel (grâce à sa participation active dans les mouvements artistiques européens de l'époque) avec la vie artistique parisienne, fut le précurseur du mouvement esthétique qui ressort des réunions du groupe Minoriste : «l'afrocubanisme». Insistant sur la nécessité artistique et sociale de cette quête esthétique, ainsi que sur les avantages que l'héritage africain offrait aux créateurs étant donné sa richesse et sa diversité, Carpentier fut en même temps l'instigateur, l'idéologue, le théoricien, le conseiller, mais aussi le «mécène» de nombreux artistes de talent de cette époque.

Dans le roman Concert baroque d'Alejo Carpentier, le personnage Francisquillo, guitariste mexicain, symbole de l'Espagne coloniale, doit mourir à La Havane et céder sa place de musicien au Noir Filomeno, porteur d'une nouvelle identité criolla ancrée dans l'héritage africain. Belle métaphore romanesque de la phrase plus synthétique utilisée par les artistes et le public d'avant-garde de l'époque comme étendard de la nouvelle révolution esthétique : «À bas la lyre, vive le bongó ${ }^{4}$. Surgissent ainsi, sous le signe de cette métaphore musicale, non seulement une musique afrocubaine, mais aussi une poésie «noire» (Emilio Ballagas [1908-1958] et Nicolas Guillén [1902-1989]) et une peinture afrocubaine (Victor Manuel [1897-1969]), appuyées dans les recherches nouvelles et scientifiques de Fernando Ortiz et la pensée culturelle globale d'Alejo Carpentier. 


\section{Amadeo Roldán ou l'afrocubanité musicale}

Le 29 novembre 1925 a lieu la création de l'Obertura sobre temas cubanos (Ouverture sur des thèmes cubains), d'Amadeo Roldán (1900-1939), par l'orchestre philharmonique, fondé deux ans auparavant. «Le $\mathrm{Xx}^{\mathrm{e}}$ siècle commence, pour la musique cubaine, en 1925, lors de la création de l'Ouverture » (Martín, cité dans Gómez, 1977, p. 46), affirme le compositeur et musicologue cubain Edgardo Martín. Pourquoi cette affirmation? C'est parce que cette œuvre sera le manifeste artistique de l'afrocubanisme, la première œuvre musicale dont la composition était animée par cette préoccupation; elle a marqué le point de départ pour l'élaboration de stratégies de création mettant en valeur les nouvelles quêtes esthétiques, pouvant être enrichies et multipliées par la suite. Cette œuvre marque également le début du symphonisme nationaliste cubain : pour la première fois, des matériaux folkloriques s'intégraient à l'écriture symphonique et, d'une certaine manière, se trouvaient «universalisés» par cette dernière. On arrivait graduellement à cette universalisation du national à laquelle aspiraient les artistes de l'époque. Rappelons que l'intérêt pour le monde afrocubain était fortement associé au besoin de mise à jour avec les tendances d'avant-garde de l'époque.

Carpentier, qui a soigneusement et régulièrement écrit sur les événements et produits culturels de cette période (comme si sa magnifique intuition d'homme de lettres et son office d'historien avaient prévu l'importance historique de ce que les artistes d'avant-garde étaient en train de concrétiser), nous dit :

Autour de 1920, la musique cubaine [...] se trouvait en franc retard par rapport à la musique universelle. Nos compositeurs se trouvaient paralysés par des formules faciles et conventionnelles, sans se préoccuper des questions d'ordre technique. [...] C'est à ce moment qu'Amadeo Roldán eut la révélation des richesses inexploitées de la musique afrocubaine. Son œuvre Obertura sobre temas cubanos apparaît pleine de trouvailles sensationnelles. Dans cette œuvre, dont les thèmes étaient extraits du vieux Cocoyé, nous entendons, pour la première fois dans notre musique symphonique, des fragments de percussion soliste confiés aux tambours, guiros, claves et gangarrias $[\ldots]^{5}$. (Carpentier, cité dans Gómez, 1977, p. 47)

Et il affirme dans un autre texte : «La transcendance de l'apport de Roldán consiste justement dans le fait qu'il s'agit d'une réalisation des aspirations les plus hautes des esprits qui voulaient l'affirmation de notre art $^{6} \gg$ (Carpentier, cité dans Gómez, 1977, p. 47).

À l'Ouverture suivent Tres pequeños poemas (Trois petits poèmes, 1926), dont la création a eu lieu en 1927 par l'orchestre philharmonique, dirigé par son fondateur, le musicien espagnol Pedro San Juan. Les trois mouvements de cette œuvre, Oriental, Pregon (Chants et cris des vendeurs ambulants) et Fiesta
5. Témoignage d'Alejo Carpentier dans Amadeo Roldán, p. 100.

6. Alejo Carpentier, "Una obra sinfónica cubana", commentaire musical lors de la création de l'Obertura. 
negra (Fête noire), constituent le premier grand succès technique et esthétique dans le travail sur des éléments afrocubains; il s'agit déjà d'une œuvre de maturité. En 1928, le chef d'orchestre Nicolai Sokolof offre sa création internationale, en Ohio, avec l'Orchestre symphonique de Cleveland.

C'est avec ces deux ouvres symphoniques, l'Ouverture et les Trois petits poèmes, que le scandale afrocubain éclate dans la société bourgeoise de l'époque; c'est à ce moment que le public se polarise en faveur de l'afrocubanisme de Roldán ou de l'indigénisme de Sanchez de Fuentes. D’où sortaient tout à coup ces rythmes violents et secs de Fiesta negra? Pourquoi les instruments populaires étaient-ils fusionnés aux instruments traditionnels de l'orchestre? Quelle était la motivation de cette danse diabolique et rituelle qui, dans les mots de Carpentier, nous donne un coup avec ses cuivres traités comme des percussions, avec ses avalanches d'accords, ses ruptures harmoniques, ses polyrythmies presque insupportables et ce silence final qui ne fait que prolonger le rituel dans notre mémoire? N'était-il pas préférable de s'aligner sur l'indigénisme puisqu'il permettait au moins de continuer à entendre des romances et des chants lyriques en gardant ainsi les vielles habitudes d'écoute? Sans doute, si l'on considère qu'il s'agit d'un public pour lequel le fait d' «être cultivé», musicalement parlant, consistait à écouter des arias connus d'opéras italiens et des chansons lyriques des compositeurs cubains en vogue, parce qu'on ne peut pas non plus parler de programmes de concert incluant les grands maîtres de la musique occidentale, mais seulement de programmes reflétant les goûts de la bourgeoisie coloniale. C'est d'ailleurs ce même mouvement d'avant-garde musicale, avec Roldán et Carpentier en tête, qui conçoit les premiers programmes de concert destinés à informer correctement les auditeurs sur les chefs-d'œuvre de la musique occidentale des siècles précédents; c'est Roldán qui offre, comme chef d'orchestre, la première audition à Cuba de la Neuvième Symphonie de Beethoven, événement qui reste dans les annales de l'orchestre de La Havane comme une date historique d'importance.

De la collaboration avec l'écrivain Alejo Carpentier naît le ballet $L a$ Rebambaramba, intitulé «ballet afrocubain». Carpentier en écrit le livret, mais aussi accompagne le compositeur dans ses travaux de terrain, véritable expérience ethnomusicologique :

On se trouvait, Amadeo et moi, à Regla, dans un prodigieux ballet ñañigo [...], qui enchânait ses figures rituelles depuis la nuit précédente. Roldán n’arrêtait pas de prendre des notes dans un cahier minuscule [...] Tout à coup, une atmosphère de malaise nous entoura. Un extraordinaire "petit diable», rouge et bleu, se cachait dans la chambre Famba (chambre sacrée). Les tambours se sont tus. Les fidèles nous regardaient avec dureté. 
- Qu'est-ce que vous écrivez là? - demanda le Illamba [maître de cérémonie des Abakuas] qui s'était aperçu de l'activité de Roldán.

- Rien... la musique... - répondit le compositeur.

- Pourquoi?

- Pour en écrire quelques danses $[\ldots]$

- Si vous ne voulez pas qu'il s'ensuive une tragédie, gardez le cahier et le crayon. Ici, personne ne vient écrire des danses.

Malgré l'invitation courtoise à nous retirer de l'endroit, nous sommes restés dans la célébration à essayer de garder en mémoire des rythmes et des mots ${ }^{7}$. (Carpentier, cité dans Gómez, 1977, p. 64)

La Rebambaramba, en version symphonique, eut un énorme succès international. Nicolas Slonimsky, compositeur et chef d'orchestre nord-américain, reconnu surtout pour son admirable labeur de diffusion et d'édition de la musique d'avant-garde des années entre 1925 et 1940, crée l'œuvre dans plusieurs pays européens :

Dans les concerts de musique panaméricaine que j'ai dirigés à Paris, Berlin et Budapest, les publics ont été si impressionnés par cette musique peu connue, mais ensorcelante, que, malgré les préjugés contre tout ce qui est transatlantique et moderne, ils ont demandé la répétition de l'un des mouvements ${ }^{8}$.

L'Orchestre symphonique de Mexico crée La Rebambaramba en 1929; l'Orchestre Straram à Paris, à la Salle Gaveau, en 1931; l'Orchestre philharmonique de Berlin, à la salle Beethoven, en 1932; l'Orchestre Hollywood Bowl, en Californie, en 1933 et l'Orchestre symphonique de la Colombie, en 1938. Même Diaghilev, en 1929, s'intéresse à la partition, mais sa mort interrompt le projet de sa création. Roldán, non sans ironie, écrit à Carpentier en 1931 : «[L]'orchestre symphonique de Mexico a joué de nouveau La Rebambaramba; la première fois c'était Chavez, maintenant Revueltas. La pauvre Rebambaramba court le danger de devenir classique... » (Roldán, cité dans Gómez, 1977, p. 68).

Suit, en 1928, l'écriture de Danza negra (Danse noire), pour un format instrumental peu commun : voix soliste, clarinette en si bémol, clarinette en la, viole I, viole II, bongó, maracas et cencerro (ces trois derniers sont des instruments de percussion utilisés dans la plupart des genres de musique traditionnelle et populaire cubaine). Danza negra, composée sur des vers du poète Luis Pales Matos, ouvre la voie à des explorations sur le plan des timbres et à des simplifications de l'écriture qui vont caractériser la dernière période de la vie créatrice de Roldán. Créée à Paris en avril 1929, sous la direction de Marius F. Gaillard, à la Salle Gaveau, cette œuvre a été intégrée dans un programme
7. Témoignage d'Alejo Carpentier dans Amadeo Roldán, p. 100.

8. Témoignage publié à Philadelphie et reproduit dans le concert hommage à la mémoire d'Amadeo Roldán, en 1939. 
9. Le Son est un des genres les plus importants de la musique populaire cubaine. Ce titre, Motivos de Son, est celui des textes du poète Nicolas Guillén, sur lesquels l'œuvre est composée.

10. Publié en 1931 à Paris, ce témoignage a été reproduit lors du concert hommage à Roldán en 1939.

11. Témoignage publié en 1929 et reproduit lors du concert hommage à Roldán en 1939. composé des Intégrales de Varèse et du Concerto de Milhaud. Ceci fait écrire à Carpentier en correspondance privée à son ami et compagnon de route:

[...] ta partition a été admirablement jouée. Quatre solistes de la Société des concerts du Conservatoire, c'est-à-dire ce qu'il y a de mieux. Gaillard t'a dirigé avec amour. Résultat, ton œuvre a été admirée par une salle comblée [...] acceptation totale du public français. (Carpentier, cité dans Gómez, 1977, p. 69)

Entre autres, deux œuvres majeures ferment la dernière période de création de Roldán : Rítmicas (Rythmiques, 1929-1930) et Motivos de Son (Motifs de $S_{o n}^{9}$, 1931). Dans la première, Roldán crée une notation spécifique pour les instruments de percussion cubains, ce qui représente un apport technique majeur pour les générations à venir. La deuxième, sur des poèmes de Nicolas Guillén, représentant indiscutable de la poésie «noire » à Cuba, a inspiré ces mots de Varèse :

[...] J'affirme qu'Amadeo Roldán est le compositeur le plus important que vous ayez à l'heure actuelle. Nous ne pouvons que déposer toute notre confiance dans ce musicien qui orchestre avec si peu d'influences extérieures et qui travaille les percussions avec une habileté surprenante, en donnant ainsi des preuves de tempérament $[\ldots]^{10}$. (Varèse, cité dans Gómez, 1977, p. 85)

Amadeo Roldán meurt en 1939, à la suite de graves problèmes de santé. Ce compositeur, en plus d'être un fondateur de la musique symphonique cubaine et un pionnier de la révolution esthétique de l'afrocubanisme, a été un admirable divulgateur de musique nouvelle, comme chef d'orchestre, et assuma la direction de la Section des Antilles de l'Association panaméricaine de compositeurs, dont la direction générale était confiée à Varèse. C'est lui qui inspire cette phrase controversée d'Hector Villa-Lobos, maintes fois citée : «Le folklore c'est moi. »

Roldán connaît le vrai chemin. Il ne pouvait pas être mieux orienté en tant que musicien d'Amérique. Dans un seul mot : il voit juste. Bientôt arrivera l'époque de création qui permettra au compositeur de dire : «Le folklore c'est moi », et l'on fera des mélodies plus authentiques que celles qui existent déjà, en les créant avec l’imagination pure. Son tempérament est indiqué pour réaliser ce labeur de surréalisme musical $[\ldots]^{11}$. (cité dans Gómez, 1977, p. 132)

\section{"La position artistique du compositeur américain"}

Henry Cowell, compositeur d'avant-garde, chercheur des musiques folkloriques, directeur des éditions New Music et l'un des fondateurs de l'Association panaméricaine de compositeurs, publie dans le livre American Composers on American Music (Stanford University Press, 1933) une lettre de Roldán sous le titre «Position artistique du compositeur américain », dans laquelle le compositeur rédige une sorte de poétique musicale. Synthétique, mais aussi passionnée, 
engagée, cette lettre est un véritable témoignage de l'esprit de l'époque. Nous réalisons, en la lisant, comment la lutte esthétique et politique menée à Cuba correspondait à une lutte plus large, panaméricaine, pour la modernité des procédés de composition, mais aussi pour la quête d'un art authentique, qui définisse l'Amérique, et qui acquiert son indépendance par rapport à l'art européen. L'intuition des résultats musicaux nouveaux, émergeant du travail sur des matériaux folkloriques uniques et spécifiques du continent américain, ainsi que sur une histoire et une pensée culturelle également spécifiques, justifie l'engagement de cette avant-garde américaine, composée des grands noms comme celui de Ives, Cowell, Chavez, Revueltas, Villa-Lobos, entre autres, et saluée avec enthousiasme par un Varèse ultramoderne et visionnaire, toujours impliqué dans les mouvements qui prenaient déjà place dans le futur et qui détenaient le pouvoir de transformer, d'innover, de créer.

Art nouveau, ressources nouvelles, encore mieux, Art américain, ressources américaines, sensibilité, formes, moyens d'expression nouveaux, américains, mais inspirés dans le plus sincère sentiment artistique, musique, arts, émotion avant tout, modernité, encore mieux, actualité dans la sensibilité et dans le langage, jamais modernisme à outrance $[\ldots]^{12}$. (cité dans Gómez, 1977, p. 167)

Sincérité dans l'expression, voici l'un des fils conducteurs de cette poétique musicale de Roldán. L’autre, c'est l'universalisation du folklore : «Étudier, développer, rendre vivant le folklore de nos pays, non pas avec le propos de construire des œuvres d'un caractère local ou national, mais avec des objectifs universalisants» (cité dans Gómez, 1977, p. 168). Originalité, mais basée sur la "pleine connaissance de tous les moyens d'expression de notre art, plus grande et plus fine simplification de la technique et des procédés, mais non à la manière de quiconque, vivant ou mort» (cité dans Gómez, 1977, p. 168). Et sur un plan plus technique, dans cette universalité originale - ou dans cette originalité universelle - l'aspect organologique détient un poids important :

L'utilisation de nos instruments autochtones, mélodiques ou de percussion, consciemment employés, non pour réussir une couleur locale facile - procédé, à mon avis, peu sérieux - mais avec l'objectif de les rendre indépendants du local [...] en les incorporant à l'ensemble des instruments actuels, pour les accompagner et parfois les remplacer, parce que les nôtres - surtout les instruments de percussion sont supérieurs en valeur rythmique et sonore aux européens. (cité dans Gómez, 1977, p. 169)

Un cri pour l'éveil d'un art américain nouveau termine ce texte d'à peine trois pages et pourtant si significatif de l'état d'esprit du moment :

Musiciens américains, propriétaires de bases mélodiques et rythmiques si variées et riches comme celles de nos pays, essayons une continentalisation de notre art,
12. Lettre à Henry Cowell, " Position de l'artiste américain ", American Composers on American Music, Stanford (CA), Stanford University Press, 1933. 
employons les moyens d'expression américains, développons, donnons de la vie à nos éléments propres, en un mot, faisons de l'art authentique américain. (cité dans Gómez, 1977, p. 169)

L'art de Roldán, étendard de l'avant-garde cubaine et américaine, montre comment les enjeux esthétiques de la création artistique de ces années aux alentours de 1930 étaient multiples, comment «modernité», « folklore », «universalité », «originalité », «maîtrise technique», «connaissance approfondie des ressources compositionnelles», «authenticité», «américanisme», "panaméricanisme », «afrocubanisme », «nationalisme», devenaient des notions complexes, pleines de significations à la fois artistiques, esthétiques, sociales, politiques, hautement émotives et profondément humaines, utilisées avec passion et fébrilité dans un moment historique où l'essor de la pensée et de l'art touche autant l'Amérique que l'Europe.

Il nous faut prendre en considération que pendant cette période historique s'enchevêtrent plusieurs événements d'importance à l'échelle internationale : la disparition de plusieurs régimes coloniaux et la conséquente sensation nouvelle de «liberté» des anciens pays colonisés; l'intensité des quêtes nationalistes menées par les nouvelles sociétés postcoloniales dans le but de se redéfinir elles-mêmes, de construire une image de la nation qui corresponde à la nouvelle situation sociale d'indépendance; les caractéristiques particulières de la période de l'entre-deux-guerres, dans laquelle l'horreur de la première guerre, l'arrivée de la paix et la crise économique mondiale de 1930 coexistent avec une fébrile activité créatrice et transformatrice qui touche autant les arts que les sciences; la nouvelle position internationale des États-Unis qui transforme le paysage mondial en déplaçant le monopole européen de l'art et des activités intellectuelles; les multiples transformations esthétiques et l'éclatement de nombreux mouvements d'avant-garde caractérisant l'art depuis le tournant du siècle, transformations radicales qui définiront le caractère du $\mathrm{xx}^{\mathrm{e}}$ siècle dans toute sa durée : un siècle de ruptures, de transformations continues, dans lequel il n'y aura pas de place à l'habitude, aux conventionnalismes gratuits, dans lequel la quête d'originalité sera parallèle à la quête du savoir et du savoir-faire; sans parler des grandes transformations sociales conduisant à la division du monde en pays socialistes et capitalistes, qui définira également la vie politique internationale du siècle au complet et qui marquera particulièrement Cuba jusqu'à nos jours.

Entre 1925 et 1940, les crises et contradictions du nouveau système socialiste n'étaient pas encore connues de tous. L'avant-garde artistique, autant en Amérique qu'en Europe, était associée, étroitement, à la pensée de gauche venant des Soviétiques. Liberté, dictature du peuple, art du peuple et pour le 
peuple, faisaient aussi partie de ce répertoire de mots et de phrases, employés avec passion, pleins d'envie de vivre et de croire aux idéaux, de transformer, de créer un monde nouveau, de changer les modes de pensée et de réussir des sociétés plus justes. Aujourd'hui, malgré le déroulement insoupçonné de l'histoire, malgré ce que les décennies suivantes ont montré comme échec politique et souvent comme injustice sociale du socialisme, malgré tout ce que nous savons maintenant, en ce début du XxI ${ }^{\mathrm{e}}$ siècle, l'idéalisme de ces artistes d'avantgarde ne cesse de nous émouvoir et de nous montrer un visage humain, rêveur, mais aussi savant, juste, clair. Le XIX ${ }^{e}$ siècle a eu ses enfants; les enfants du XX siècle seront les rêveurs passionnés de cette génération de l'entre-deux-guerres, dans laquelle il ne manque pas de romantisme ni d'idéaux de justice, de liberté et de fraternité.

Les noms de Roldán et d'autres compositeurs de cette période, comme Alejandro García Caturla (1906-1940), restent dans l'histoire de la musique cubaine comme de véritables personnages romanesques, de grands idéalistes et des lutteurs infatigables, avec des destins tragiques (la mort de Roldán, à l'âge de 39 ans, est survenue juste un an avant l'assassinat de Caturla, à 34 ans, à cause de ses idéaux de justice), mais aussi de vrais chercheurs de vérité. Et surtout, ils sont les instigateurs de toute une lignée de compositeurs d'avant-garde qui pendant le $\mathrm{xx}^{\mathrm{e}}$ siècle ont suivi leurs exemple, suscitant de décennie en décennie de nouvelles mises à jour par rapport à la modernité et à l'originalité des procédés de composition et des intentions esthétiques. Ces compositeurs des générations ultérieures, parmi lesquels nous trouvons les noms de Harold Gramatges et Leo Brouwer, pour ne citer que les plus connus en dehors de Cuba, ont revu continuellement la pertinence de l'utilisation des matériaux folkloriques, poursuivant la recherche des fusions organologiques entre les instruments européens et les instruments traditionnels cubains, perpétuant ainsi l'entrelacement entre la musique classique et la musique folklorique.

Remarquons aussi l'importance du mouvement afrocubain en général dans l'évolution des sciences comme l'ethnomusicologie, l'ethnologie générale et l'anthropologie, qui, à partir des années 1930, ont vu un développement toujours croissant à Cuba, et dont l'application à la recherche et à la création musicales n'a fait que s'intensifier avec les écoles de pensée qui ont perpétué l'héritage de Fernando Ortiz.

Il est donc vrai que le $\mathrm{xx}^{\mathrm{e}}$ siècle de la musique cubaine naît dans cette décennie «critique», comme l'affirme le musicologue Edgardo Martín, puisque ce sont les caractéristiques qui la définissent encore aujourd'hui qui ressortent de ce magnifique point de départ de l'afrocubanisme. La poétique de Roldán, exprimée dans la Lettre à Henry Cowell, sert à définir dans une image synthétique toute la 
création musicale cubaine jusqu’à nos jours, comme une spirale qui revient, avec des concepts nouveaux et de nouveaux procédés, mais toujours avec la même quête d'identité et d'universalité.

\section{B I B L I O G RAPH IE}

Carpentier, Alejo (2004), La música en Cuba, La Havane, Editorial Letras cubanas. Carpentier, Alejo (2001), Testimonios, La Havane, Editorial Letras cubanas.

Chang, Leiling (2002), Métissages et résonances. Essais sur la musique et la littérature cubaines, Paris, L’Harmattan.

Enríquez, Maria Antonieta (1998), Alejandro García Caturla, La Havane, Ediciones Union.

Gómez, Zoila (1977), Amadeo Roldán, La Havane, Editorial Arte y Literatura.

León, Argeliers (1984), Del canto y el tiempo. La Havane, Editorial Letras cubanas. 